Volume 9, No.5, September - October 2020

International Journal of Advanced Trends in Computer Science and Engineering

Available Online at http://www.warse.org/IJATCSE/static/pdf/file/ijatcse35952020.pdf

https://doi.org/10.30534/ijatcse/2020/35952020

\title{
Applying AHP to Formulate Weighted-Sum Goal Programming in Solving the RNP problem in WSNs
}

\author{
Mohammed Alreshoodi $^{1}$, Bader Alshaqqawi ${ }^{2}$, Sardar Anisul Haque ${ }^{3}$, Ibrahim S. Alsukayti ${ }^{4}$ \\ ${ }^{1}$ Department of Applied Science, Unizah Community College, Qassim University, Buraydah, Saudi Arabia, \\ MO.ALRESHOODI@qu.edu.sa \\ ${ }^{2}$ Department of Mathematics, Collage of Science, Qassim University, Buraydah, Saudi Arabia, \\ bshkaoy@qu.edu.sa \\ ${ }^{3}$ Department of Mathematics and Computer Science, Alcorn State University, Lorman, Mississippi, USA, \\ sahaque@alcorn.edu \\ ${ }^{4}$ Department of Computer Science, Collage of Computer, Qassim University, Buraydah, Saudi Arabia, \\ skiety@qu.edu.sa
}

\begin{abstract}
Effective optimization of the Relay Node Placement (RNP) problem is a key to realize efficient deployment of WSNs. Inefficiently addressing such a design problem would adversely impact the overall performance of WSNs in terms of many aspects including energy efficiency. In this paper, the RNP problem is addressed as a multi-objective optimization problem for improving network coverage, minimizing energy consumption, and maintaining low deployment cost. We considered unconstrained deployment of energy-harvesting RNs in pre-established stationary WSNs. The RNP problem was approached in this work using a novel Goal Programming (GP)-driven metaheuristic approach. It is based on formulating the optimization problem using the weighted-sum GP model and then applying the Analytical Hierarchy Process (AHP) method for effective assignment of the relative weights to the decision variables. To efficiently solve the GP-formulated optimization problem, we developed an enhanced GA model integrating a greedy technique. We focused our evaluation on experimentally investigating how good the AHP technique is in generating the parameters for the GP model considering the RNP problem. As the evaluation results indicated, our GP-driven greedy-enhanced GP optimization model provided a highly comparable solution quality to that of the multiobjective GA model. It even outperformed the multiobjective GA model in those RNP problems with relatively small scale setups.
\end{abstract}

Key words: Wireless Sensor Network, Goal Programming, Analytical Hierarchy Process, Metaheuristics.

\section{INTRODUCTION}

Wireless Sensor Networks (WSNs) have emerged as one of the key established technologies for a wide range of applications in many domains such as industry, healthcare, surveillance, agriculture, and utilities. In such applications, a number of small-sized sensor nodes of limited computing, energy, and communication resources are placed over varying-scale deployment areas for long-term operation. They typically participate in sensing surrounding environments and communicating captured sensor data using low-power wireless communication technologies.

Realizing efficient deployment of WSNs is challenging unless careful design of different networking aspects is accomplished. One of the key design principles in WSNs is the deployment of additional nodes, the Relay Nodes (RNs), in a higher tier of the network architecture to interconnect and relay the traffic of the sensor nodes. These are mains-powered or energy-harvesting networking devices of higher computing capabilities. This is important to alleviate the strict limitations of the resource-limited sensor nodes and improve network lifetime. However, there is a critical need to identify the optimal number and best locations of RNs to be deployed for efficiently covering the network area with cost-effective and energy-efficient deployment. Inefficiently addressing such a design problem would impact the overall performance of WSNs in terms of energy efficiency, deployment cost, and network coverage.

This is known as the Relay Node Placement (RNP) problem which is one of the critical design problems in WSNs. It is a Non-Deterministic Polynomial-time (NP)-hard optimization problem. It can be efficiently addressed to successfully meet design requirements and achieve multiple optimization objectives. A number of conflicting objectives can be formulated in single problem definition. These could include network connectivity, area coverage, energy efficiency, overall performance, and cost effectiveness.

This would entail the need for metaheuristics to have a polynomial time methods providing near optimal solutions. However, deterministic approaches can also be considered to establish the optimization solution. In general, establishing an efficient solution to this kind of problem would revive the potentiality of improving WSN deployment. The outcome of this work will contribute to the acceleration of WSN development for a wide range of applications. 
In this paper, the RNP problem is addressed as a multi-objective optimization problem considering unconstrained deployment of energy-harvesting RNs in pre-established stationary WSNs. Different optimization criteria were considered in our objective functions for improving network coverage, minimizing energy consumption, and maintaining deployment cost. To efficiently address such an optimization problem, we propose the development of a novel Goal Programming (GP)-based metaheuristic approach. It is based on weighted-sum GP formulation of the RNP problem to determine each objective by certain goals estimated based on realistic setups of different WSN applications. As there are conflicting optimization objectives, the Analytical Hierarchy Process (AHP) method is integrated to realize an efficient approach for successfully weighting the objectives. An enhanced GA model integrating a greedy technique is then developed to solve the optimization problem which incorporates the GP goals as constraints in addition to the AHP weight configurations. In addition, this work will present a more practical contribution with the implementation of the proposed approach. As indicated by the experimental results, the solution quality provided by our GP-driven greedy-enhanced GP optimization model was highly comparable to that of the multiobjective GA model. Moreover, as the RNP problem scaled down, it achieved better performance than the multiobjective GA model considering varying sets of preferences to the considered objectives.

The rest of the paper is structured as follows: Section2 presents related research efforts. In Section 3 and 4, we present the problem formulation and search space processing, respectively. Section 5 describes the proposed optimization approach. In Section 6, the evaluation setup is described and the experimental results are discussed. The conclusion is presented in Section 7.

\section{RELATED WORK}

WSNs are typically characterized by their strictly limited resources considering many aspects such as computing capacity, communication capabilities, and power supply. Therefore, there have been significant research efforts devoted to address related optimization problems in WSN. Examples are energy efficiency, network routing, security, clustering, and mobility support [1]-[5]. Considering the complete WSN deployment cycle which goes through different phases, most of these problems are considered in the post-deployment and redeployment phases [6]. Other WSN problems including the RNP problem need to be addressed during the pre-deployment and deployment phases. The RNP problem is one of the key design problems in WSNs which has been addressed as a challenging NP-Hard optimization problem.

A number of optimization techniques have been adopted to address the different optimization problems in WSNs. It has been a common practice to approach such a complex problem using metaheuristics [7]. However, deterministic algorithms such as GP [8] can also be of great use to provide efficient optimal solutions using simple formulation. It provides a feasible analytical approach providing a mathematical programming-based classical scalarization method [1].

GP was originally proposed by Charnes et al. in [9] and then further extended in [10]-[12]. It is one of the early developed methods to support multi-objective optimization problems. In contrast to other conventional optimization methods, GP is based on a flexible model to reflect real operation conditions using simple formulation. Thus, it provides an effective approach for enabling linear and non-linear programming to optimize multi-objective optimization problems in a simple and flexible manner. GP is based on having a user-specified target value assigned for each objective and incorporated into the problem definition as additional constraints. The main working principle of GP is then based on maximizing the achievement of the corresponding targets simultaneously. This is based on using an achievement function to minimize the total of the absolute deviations from the target values of the conflicting objective. Thus, the deviations determine the objectives of the optimization problem. There are different GP approaches including Archimedean (weighted-sum) GP, lexicographic (preemptive) GP, multichoice GP, and Chebyshev (MINMAX) GP [13]. For example, the Archimedean GP model is based on minimizing the weighted sum of the deviations whereas the maximum deviation is minimized in the Chebyshev GP model.

Therefore, GP has been successfully applied to a variety of multi-objective optimization problems in the domain of WSNs. For example, the GP algorithm was adopted in [14] to address arbitrary static network deployment in the context of Wireless Multimedia Sensor Networks (WMSNs). The focus was on optimizing two conflicting objectives: maximizing network lifetime while improving the overall network throughput to meet the requirements of multimedia services in WMSNs applications. The evaluation results showed how applicable and feasible the goal programming technique to meet the network performance and lifetime requirements of real-problems. In [15], a GP-based approach was proposed to address energy-efficient event recognition in WSNs. This was based on finding the optimal number of active sensor nodes to achieve satisfactory recognition performance while ensuring lower energy consumption. Therefore, two conflicting objectives were considered: improving pattern recognition success and minimizing energy consumption.

In [16], a GP-based approach was proposed to optimize a Distributed Denial of Service (DDoS) attack detection scheme for WSNs. The optimization was achieved by addressing the optimal configuration of a network parameter known as application aspect ratio based on two conflicting objects: attack detection rate and energy decay rate. The empirical analysis of the proposed GP-based approach indicated its effectiveness in finding the optimal options for the detection scheme. In [17], GP was adopted for addressing the negative effects of hidden terminals in Wireless Mesh Sensor Networks (WMSNs) running the IEEE 802.15.5 standard, in particular, its Asynchronous Energy Saving (ASES) mode. 
This phenomenon causes message collisions and leads to increasing retransmissions and packet loss across the network. As a result, the overall performance of the network would degrade and energy consumption would increase. The hidden terminals problem was formulated as a multi-objective optimization problem defining two objectives: maximizing network lifetime and minimizing the aggregate message collision time. Such a multi-objective formulation was then addressed using a GP-based algorithm.

GP has also been considered for other optimization problems in the general field of computer networking. In [18], a GP-based approach was proposed to address optimal network topology design problems in a remote patient monitoring system. The approach integrated a GP variant, namely the multi-choice GP, with a S-shaped penalty method, arbitrary piece- wise linear utility functions, and trapezoidal utility functions. Qualitative and quantitative objectives were considered to address the establishment of a complete topology network to increase citizen satisfaction under a limited budget. In [19], the optimization problem of Quality of Service (QoS)-oriented resource allocation in energy harvesting systems was addressed. The focus was on limiting the impact of uncertainty of harvested energy on QoS provisioning. GP was adopted to formulate the problem towards minimizing user dissatisfaction while allocating resources effectively. Accordingly, two schemes were developed for this problem to address offline and online resource allocation using a dual decomposition algorithm and dynamic programming, respectively.

There have also been some attempts to address effective network deployment using approaches that incorporate GP-based formulation into evolutionary algorithms such as the Genetic Algorithm (GA). In [20], optimized deployment of wireless access points was addressed towards optimal wireless LAN design. An integrated optimization approach integrating a GP-based problem modelling and a GA scheme was proposed. GP was adopted for identifying the target subjects of multiple objectives. These include minimizing deployment cost, maximizing network coverage, balancing access load, and reducing interference. The results demonstrated the feasibility and stability of the proposed approach to optimize access points deployment considering large-scale problems. In [21], the focus was on fault-tolerant $\mathrm{RN}$ deployment in WSNs to minimize the number of RNs while maximizing the connectivity among the RNs and sensor nodes. Such an optimization problem was formulated using Linear Programming (LP) to identify certain connectivity constraints. The GA was then incorporated to find the least number of deployment positions that ensure k-connectivity. Some researchers have applied Analytic Hierarchy Process (AHP) [22] in different WSNs optimization problems [23]-[25]. In this paper, we propose a more effective approach to formulate the RNP problem using the weighted-sum GP model and apply an enhanced GA scheme to optimize multiple objectives. These include minimizing deployment cost, maximizing network coverage, and improving energy efficiency. Moreover, applying the AHP provides an effective approach to determine the relative weights for decision variables in the optimization problem. It enables effective logical computation of the different weights assigned to each objective. In order to further enhance our optimization approach, we apply AHP to develop a more effective weighted-sum GP model. To the best of our knowledge, our proposal is the first to consider AHP for calculating weights in a weighted-sum GP model. Though we apply this model to solve the RNP problem, we expect that other multi-objective problems can be solved effectively using this model.

\section{GP-DRIVEN FORMULATION FOR THE RNP PROBLEM}

We construct a weighted-sum goal programming formulation [26] for our RNPP problem considering (i) the number of RNs, (ii) the distance from each sensor node to its nearest RN, and (iii) the energy consumed by each sensor node. We express each of these properties as an objective function as follows:

(i) Cost: the cost associated with the procurement, installment, and maintenance of RNs that are suggested in a candidate solution. Let, $\max$ be an integer that we assume to be an upper bound for the expected number of RNs. We obtain this number by a greedy algorithm described in Section 4. For any candidate solution, let $r_{\text {candidate }}$ be the number of RNs to solve a RNP problem, where $r_{\text {candidate }} \leq \max$. We compute cost as the ratio between $r_{\text {candidate }}$ and max. This is a minimization objective.

$$
f_{1}=\frac{r_{\operatorname{atandiasix}}}{\max }
$$

As we mentioned above, we computed $\max$ by a greedy algorithm. We found it as a near optimal number of relay nodes experimentally. We expect that if $\mathrm{r}_{\text {candidate }} \geq 0.8 \max$ then it should be very promising. So our corresponding goal is described below.

$$
\mathrm{p}_{\text {cost }}=\mathrm{f}_{1}-0.80
$$

(ii) Coverage: the fraction of sensor nodes that can communicate with at least one RN. Let $s^{\prime}$ be the number of sensor nodes that can communicate with at least any RN for a given solution. This is a maximization objective.

$$
f=\frac{z}{z}
$$

We set a threshold value of 0.97 on the expected lower bound on the value of $f_{2}$. So the corresponding goal is described below.

$$
\mathrm{n}_{\text {coverage }}=0.97-\mathrm{f}_{2}
$$

(iii) Energy: Let the maximum distance possible for a sensor to reach the nearest $\mathrm{RN}$ is limited by $c$ units. Let the sum of distances traveled by the sensor nodes, which has the nearest RN in c units, be Distance. We compute energy as the ratio between Distance and $\mathrm{s}^{*} \mathrm{c}$. This is a minimization objective.

$$
f=\frac{\text { Distance }}{\sec }
$$

We set a threshold value of 0.50 on the expected lower bound on the value of $f_{3}$. So the corresponding goal is described below.

$$
p_{\text {energy }}=f_{3}-0.5
$$




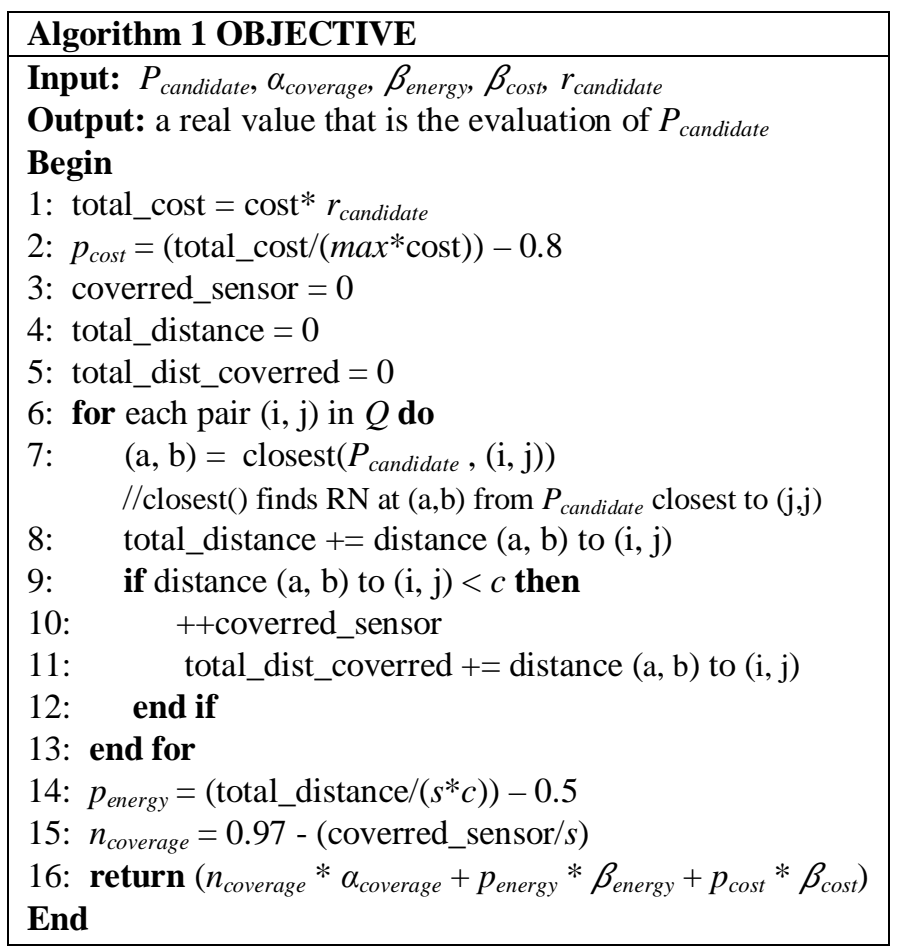

So the objective function $F$ of our weighted-sum goal programming model is formulated as follows:

$$
F=\alpha_{\text {coverage }} n_{\text {coverage }}+\beta_{\text {energy }} p_{\text {energy }}+\beta_{\text {cost }} p_{\text {cost }}
$$

where the parameters $\alpha_{\text {coverage }}, \quad \beta_{\text {energy }}, \quad \beta_{\text {cost }}$ are the corresponding weight factors. These will be computed by using AHP. The choice of these parameters can be determined by AHP based on users' perspectives and preferences.

After that, we use an enhanced GA algorithm to minimize F. We understand that there exist a number of powerful meta-heuristic algorithms in the literature and complex mathematical models to explain RNP problems. However, we focus on our primary aim to investigate how good AHP techniques are in generating the parameters in GP for RNP problems. Our enhanced approach is based on integrating GA with a greedy algorithm to effectively determine the upper bound on the number of RNs and locate the hotspots in the search space. The technique of integrating a greedy algorithm with a simple meta-heuristic algorithm can be equally applicable to other powerful meta-heuristic algorithms. We did our experimentations on a very simple mathematical model to represent the RNP problem. We assume that a near optimal solution suggested by our solution to this simple model should be also a near-optimal solution to a relatively complex model. Algorithm 1 OBJECTIVE describes our weighted-sum GP approach.

\section{SEARCH SPACE PROCESSING}

The mathematical model of our RNP problem is completed by preprocessing the search spaces, which is described in subsection 4.1. Accordingly, the locations of both sensor and relay nodes are redefined, and this procedure is explained in subsection 4.2.

\subsection{Search Space Preprocessing}

Input to RNPP is a list of sensors, each of which is described by a point. The list of points is drawn from a 2-D space. We will consider this as the search space associated with the given RNP problem. The unit of our search space is arbitrary. We limit this search space by computing both maximum and minimum values in both $\mathrm{X}$ and $\mathrm{Y}$ direction. The solution to RNP problem is also a set of points, each of which describes the location of a RN. It should be noted that both input and output data are supposed to be continuous variables. This makes a RNP problem computationally hard as the optimal points can have arbitrary precision during the optimization process. To overcome this situation, we build a mathematical model of our problem which deals with only integers while describing the locations of both sensors and relay nodes.

Let $d$ be a small value such that if a sensor or relay node's location is moved $d$ units in $\mathrm{X}$ or $\mathrm{Y}$ direction, it causes negligible effect on our near-optimal solution, and the distance between any two sensor nodes is more than $d$. Let $\left(\mathrm{x}_{\min }, \mathrm{y}_{\min }\right),\left(\mathrm{x}_{\max }, \mathrm{y}_{\min }\right),\left(\mathrm{x}_{\max }, \mathrm{y}_{\max }\right)$, and $\left(\mathrm{x}_{\min }, \mathrm{y}_{\max }\right)$ be the four corners of the smallest rectangle of our search space such that all input points (locations of sensors) are inside of it and the distance from any sensor to any side of the rectangle is more than $d$. For simplicity, let the length of each side of the rectangle be a multiple of $d$. We divide the rectangle by ( ( $\mathrm{x}_{\max }$ $\left.\left.-\mathrm{x}_{\min }\right) / \mathrm{d}-1\right)$ straight lines that are parallel to $\mathrm{Y}$ and $\left(\left(\mathrm{y}_{\max }-\right.\right.$ $\left.y_{\min }\right) / d-1$ ) straight lines that are parallel to $X$. These two groups of straight lines will divide the rectangle in such a way that it will have $\left(\left(\mathrm{x}_{\max }-\mathrm{x}_{\min }\right) / \mathrm{d}\right) *\left(\left(\mathrm{y}_{\max }-\mathrm{y}_{\min }\right) / \mathrm{d}\right)$ number of squares, each of the squares has area $d^{2}$ units. This makes the discretization of any point in the search space straight forward, which can be described as follows: Let $\left(\mathrm{x}_{\mathrm{i}}, \mathrm{y}_{\mathrm{i}}\right)$ be a point in our search space. This point is located in the square marked by a pair of two integers (floor $\left(\mathrm{x}_{\mathrm{i}} / d\right)$, floor $\left(\mathrm{y}_{\mathrm{i}} / d\right)$ ).

The above procedure redefines not only the sensor nodes' positions but also the search space itself. Our new search space becomes a grid having ceil $\left(\left(\mathrm{x}_{\max }-\mathrm{x}_{\min }\right) / d\right)$ and ceil( $\left(\mathrm{y}_{\max }-\mathrm{y}_{\min }\right) / d$ ) squares in $\mathrm{X}$ and $\mathrm{Y}$ direction respectively. In this new search space, our computation involves integers only considering the locations of sensor and relay nodes.

The output to our optimization approach will be a set of pairs of integers. Each pair defines a particular square in the search space, into which we can place a RN. The actual position of the RN should not affect our near-optimal solution according to the definition of $d$.

\subsection{Redefinition of Points in Search Space Domain}

Let $m=\operatorname{ceil}\left(\left(\mathrm{x}_{\max }-\mathrm{x}_{\min }\right) / d\right)$ and $n=\operatorname{ceil}\left(\left(\mathrm{y}_{\max }-\mathrm{y}_{\min }\right) / d\right)$. Now the sensor nodes can be described by $S$, a zero-one matrix of order $m$ and $n$, where

$$
S[i][j]=\left\{\begin{array}{l}
1, \text { if there is a sensor node in }(i, j) \text { square, } \\
0, \text { Otherwise. }
\end{array}\right.
$$

We define $s$ as the total number of sensor nodes. Clearly, $\Sigma_{\text {Datemos } j \mathrm{en}} 5[i][j]=s$.

$Q$, is a list of $s$ unique pairs of integers, such that each pair $(i, j)$ describes a square in which a particular sensor node is located, where $S[\mathrm{i}][\mathrm{j}]=1$. 
Let $w$ be a value such that a sensor node's signal can travel at best. Let $c=$ floor $(w / d)$. It means that a sensor node can communicate with a $\mathrm{RN}$ if and only if the distance between them is at most $c$ square units. In other words, if the nearest $\mathrm{RN}$ of a sensor node is $c$ squares away from it in both $\mathrm{X}$ or $\mathrm{Y}$ direction, the sensor node cannot communicate with it.

We also define $R$ as a zero-one matrix of order $m$ and $n$ to store the positions of RNs, as follows:

$$
R[i][j]=\left\{\begin{array}{l}
1, \text { if there is a } R N \text { in }(i, j) \text { square, } \\
0, \text { Otherwise. }
\end{array}\right.
$$

Let $r$ be the total number of RNs, which we expect to find by our solution. Clearly, $\sum_{0 \leq i s m, 0 \leq j e m} R[i][j]=r$.

The output of our solution is $P$, a list of $r$ unique pairs of integers, where each pair $(i, j)$ describes a square in our search space in the 2-D space, where R[i][j] $=1$. We consider $P$ as a near-optimal solution to the RNP problem.

\section{ENHANCED METAHEURISTIC OPTIMIZATION}

After formulating our RNP problem using the weighted-sum GP model, our proposed solution then proceeds into three stages, as follows:

(i) we compute $\alpha_{\text {coverage }}, \beta_{\text {energy }}, \beta_{\text {cost }}$ using AHP.

(ii) we apply a greedy algorithm to find out some squares on the search space in which we observe signals from a maximum number of sensor nodes. In other words, the greedy algorithm tries to place relay nodes to reduce the energy consumption by sensor nodes. As the greedy algorithm is used to accelerate the convergence in the meta-heuristic algorithms, we are not looking for any complete solution from it.

(iii) we instrument and execute the meta-heuristic algorithm to solve the weighted-sum GP with the help of that list of squares returned from the greedy algorithm.

The following subsections provide descriptions of each of these three stages.

\subsection{Analytical Hierarchy Process}

AHP is one of multi-criteria decision making method that was originally developed by Prof. Thomas L. Saaty [20]. It is a method to derive ratio scales from paired comparisons. The input to AHP method can be obtained by answering the following questions. These questions can be asked to the people who want to work with our RNP problem can be:

a) How much importance do you want to put on cost over network coverage?

b) How much importance do you want to put on cost over energy consumption?

c) How much importance do you want to put on energy consumption over network coverage?

The answer should be in any of the following integer values between 1 to 9 or its reciprocal as follows

a) Equally important (equivalent numeric value is 1)

b) Moderately important (equivalent numeric value is 3 )

c) Strongly important (equivalent numeric value is 5)

d) Very strongly important (equivalent numeric value is 7)

e) Extremely important (equivalent numeric value is 9)
Any even value (specifically the values: $2,4,6,8$ ) in the above list is treated as an intermediary value. For example, let cost be moderately important (numerical value 3 ) than energy. Then the importance of energy over cost can be expressed by a numeric value of $1 / 3$.

Once all the comparisons among our objective criterions are accomplished, we can apply AHP techniques to compute normalized (between $[0,1]$ ) principal Eigenvector. The corresponding values will be taken as weights in Algorithm 1.

\subsection{The Greedy Algorithm}

We need to list the following notations in order to explain the working principle of our greedy algorithm.

Let $R_{\text {greedy }}$ be a zero-one matrix of order $\mathrm{m}$ and $\mathrm{n}$, where:

$$
R_{\mathrm{ges}}[i][j]= \begin{cases}1, & \text { if GREEDY puts a RN in square }(i, j) \\ 0, & \text { otherwise. }\end{cases}
$$

Let $r_{\text {greedy }}$ be the total number of RNs that our greedy algorithm suggests to be placed across the search space. It is clear that $\Sigma_{0 \text { sicm, } 05 j \times n} R_{\text {greedy }}[i][j]=r_{\text {greedy }}$.

In the greedy algorithm, we assume that each sensor node can connect to a $\mathrm{RN}$ if the distance between them is equal or smaller than $c_{\text {greedy }}$ squares. $c_{\text {greedy }}$ is an artificial value such that $c_{\text {greedy }}>c$. It is expected that $r_{\text {greedy }}$ is inversely proportional to the value of $c_{\text {greedy. }}$.

Our greedy algorithm is explained with the ComputingInfluence and GREEDY algorithm as presented in Algorithm 2 and 3, respectively. The input to ComputingInfluence is a set of sensor nodes, $G$. The output of this algorithm is $I$, an integer matrix of order $m$ and $n$. I[i][j] is the number of sensor nodes from $G$ whose signal can be reached at square $(i, j)$ assuming that the signal from each sensor node can be propagated $c_{\text {greedy }}$ squares in all directions. The GREEDY algorithm is an iterative algorithm. The input to this algorithm is $Q$, which is the set of all sensor nodes. In each iteration, it computes $I$ using ComputingINfluence. It then finds the maximum value in $I$. Let $I[\mathrm{i}][\mathrm{j}]$ contains maximum value, it then updates with $R[\mathrm{i}][\mathrm{j}]=1$ and deletes the sensor nodes which could be served by placing a RN at (i, j) before starting the next iteration (if any).

\begin{tabular}{|l|}
\hline Algorithm 2 ComputingInfluence \\
\hline Input: Set $G$ \\
Output: Matrix $I$ of order $m$ and $n$ \\
Begin \\
1: Create a zero matrix $I$ of order $m$ and $n$ \\
2: for each sensor node $g$ in $G$; where $g$ is at $(\mathrm{i}, \mathrm{j})$ do \\
$3: \quad$ for each square $(\mathrm{x}, \mathrm{y})$ in $I$ do \\
$4: \quad \quad$ if distance from $(\mathrm{i}, \mathrm{j})$ to $(\mathrm{x}, \mathrm{y}) \leq \mathrm{c}_{\text {greedy }}$ then \\
5: $\quad I[\mathrm{x}][\mathrm{y}]=I[\mathrm{x}][[\mathrm{y}]+1$ \\
6: $\quad$ end if \\
7: end for \\
8: end for \\
9: return $I$ \\
End \\
\hline
\end{tabular}




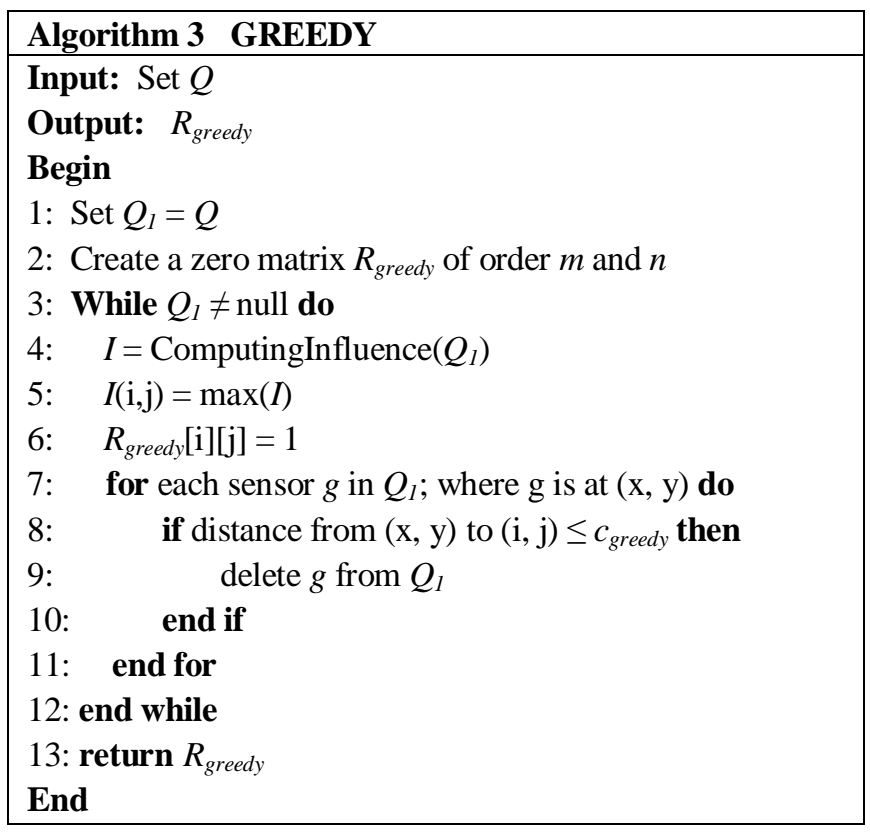

\subsection{The Meta-Heuristic Algorithm}

We developed an effective optimization approach by having a greedy algorithm integrated with a meta-heuristic algorithm. Although this can be equally applicable to other powerful meta-heuristic algorithms, we adopted the GA in this study. The GA is executed once the greedy algorithm completes its execution. We achieve two very important information from the greedy algorithm:

(i) the approximate number of $\mathrm{RN}$ that can be used to serve all sensor nodes

(ii) the squares in the search space where sensor nodes are more likely to be placed.

These two outputs are then used to effectively configure the GA to solve our RNP problem.

From the GA, we are expecting two kinds of RNs, as follows:

(i) $\mathrm{r}_{\text {firstKind }}$ : First Kind RNs are the relay nodes that are being tried to place near to the squares suggested by the greedy algorithm. To describe this kind of RNs, we need to introduce another artificial communication range $c_{\text {outerCircle }}$, where $c_{\text {greedy }}>c_{\text {outerCircle }}>c$. In total, GA will try to place $r_{\text {greedy }}$ number of RNs of this kind. The range of each of the RN's coordinate values is by both adding and subtracting $c_{\text {greedy }}$ with the corresponding coordinate positions from greedy algorithm. The main purpose of these RNs is to increase the coverage metric. During the convergence phase of GA, a RN will be considered for fitness value if it is, by random choice, not more than $c_{\text {outerCircle }}$ away from the position suggested by the greedy algorithm. This technique gives us the flexibility for our GA on the number of RNs. We use $r_{\text {first Kind }}$, to represent the total number of RNs of first kind.

(ii) $r_{\text {secondKind }}$ : Second Kind RNs are the RNs that are being tried to place anywhere in the search space. To describe this kind of RNs, we need to introduce $m_{\text {outerBox }}, n_{\text {outerBox }}$, where $m_{\text {outerBox }}>m$ and $n_{\text {outerBox }}>n$. The GA will try to place a number of RNs of second kind. The range of coordinates of each $\mathrm{RN}$ is defined as $\left(0, m_{\text {outerBox }}\right)$ and $\left(0, n_{\text {outerBox }}\right)$. The main purpose of these RNs is to get rid of local optima that might be caused by those of first kind. The number of RNs of second kind will depend on the complexity of RNPP. If the solution from the GA algorithm is not satisfactory considering energy consumption and network coverage, it can be increased. During the convergence phase of the GA, a $\mathrm{RN}$ will be considered for fitness value if it is, by random choice, placed in the legal boundary of the search space. This technique gives us the flexibility for our GA on the number of RNs. We use $r_{\text {secondKind }}$, to represent the total number of RNs of second kind.

\section{EVALUATION}

\subsection{Evaluation Setup}

Effective evaluation of the proposed approach was carried out using an experimental dataset which represents multiple RNP problem instances with complex and challenging WSN setups. In all the instances, we considered unconstrained deployment of RNs in a stationary WSN implemented with a moderate number of sensor nodes. Sensor nodes were considered to be deployed with a random distribution in a square-shaped area. We set the same communication range and $d$ for the sensor nodes and RNs to be $30 \mathrm{~m}$ and $3 \mathrm{~m}$, respectively, for all the experiments. The values of the weight factors, $\alpha_{\text {coverage }}, \beta_{\text {energy }}, \beta_{\text {cost }}$, were computed using the AHP method. Different sets of weights were generated assuming different preferences for varying WSN applications. For example, the network coverage was significantly prioritized in some of the setups thus AHP gave a weight value to this objective higher than those assigned to the energy consumption and cost objectives. In other setups, minimizing energy consumption was prioritized over the other objectives. The main details for the setup of each experiment are given in Table 1.

The implementation and evaluation were accomplished using MATLAB R2018b over a system with an Intel Core i5, 2.5 $\mathrm{GHz}$ CPU, 4 GB RAM, and Mac OS. For all the experiments, the basic parameters of the GA were configured similarly. However, a different initial population was considered in each experiment according to the area size of the given problem. The number of RNs is provided by the greedy algorithm as an input to the GA process. The crossover rate was set to $8 \%$. The stall generation and function tolerance value were configured to be 50 and $1 \mathrm{e}-3$, respectively.

To mitigate the randomness effect in such optimization processes, each experiment was run for at least 10 times independently. Then, the collected results were averaged for each experiment. In all the experiments, a confidence level of $95 \%$ was considered.

To demonstrate its efficiency, a comparison was established between the proposed GP-driven GA approach and the pareto front generated by a multi-objective GA model. The comparison was carried out considering the solution quality 
which was indicated by the values of the objectives: network coverage, energy consumption, and cost. We configured the multi-objective GA implementation provided in MATLAB to find the pareto front for the given RNP problem. The obtained pareto fronts for all the three instances are presented in Figure 1-3. This MATLAB function is also configured in such a way that it can take advantage of our greedy algorithm. The objective function is developed for our multi-objective genetic algorithm in such a way that all three priorities are given equal importance.

Table 1: Details of the Experimental Setups

\begin{tabular}{|c|c|c|c|c|c|c|}
\hline Exp. & Size $\left(\mathbf{m}^{\mathbf{2}}\right)$ & $\boldsymbol{s}$ & $\boldsymbol{r}$ & $\boldsymbol{\alpha}_{\text {coverage }}$ & $\boldsymbol{\beta}_{\text {energy }}$ & $\boldsymbol{\beta}_{\text {cost }}$ \\
\hline Exp1 & $500 \times 500$ & 250 & 50 & 0.59 & 0.33 & 0.08 \\
\hline Exp2 & $500 \times 500$ & 250 & 50 & 0.33 & 0.08 & 0.59 \\
\hline Exp3 & $500 \times 500$ & 250 & 50 & 0.08 & 0.59 & 0.33 \\
\hline Exp4 & $600 \times 600$ & 300 & 63 & 0.59 & 0.33 & 0.08 \\
\hline Exp5 & $600 \times 600$ & 300 & 63 & 0.33 & 0.08 & 0.59 \\
\hline Exp6 & $600 \times 600$ & 300 & 63 & 0.08 & 0.59 & 0.33 \\
\hline Exp7 & $800 \times 800$ & 400 & 91 & 0.59 & 0.33 & 0.08 \\
\hline Exp8 & $800 \times 800$ & 400 & 91 & 0.33 & 0.08 & 0.59 \\
\hline Exp9 & $800 \times 800$ & 400 & 91 & 0.08 & 0.59 & 0.33 \\
\hline
\end{tabular}

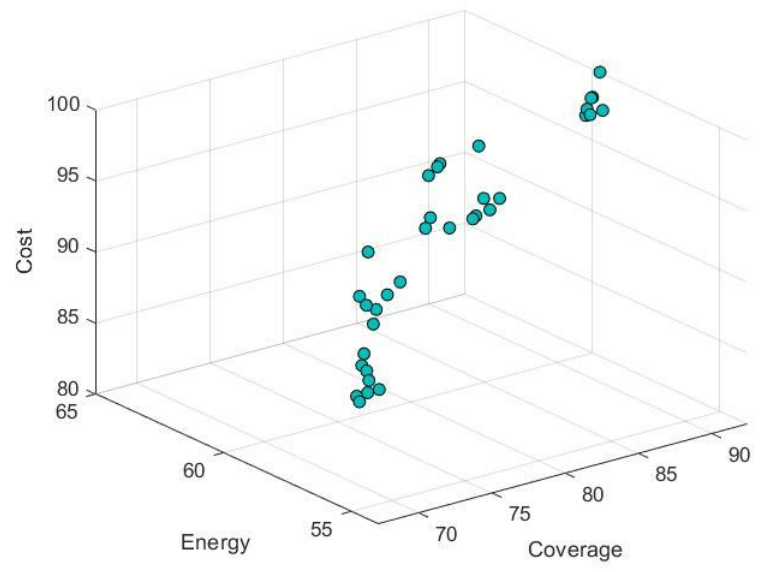

Figure 1: The Pareto Front for the Instance of size $500 \times 500 \mathrm{~m}^{2}$

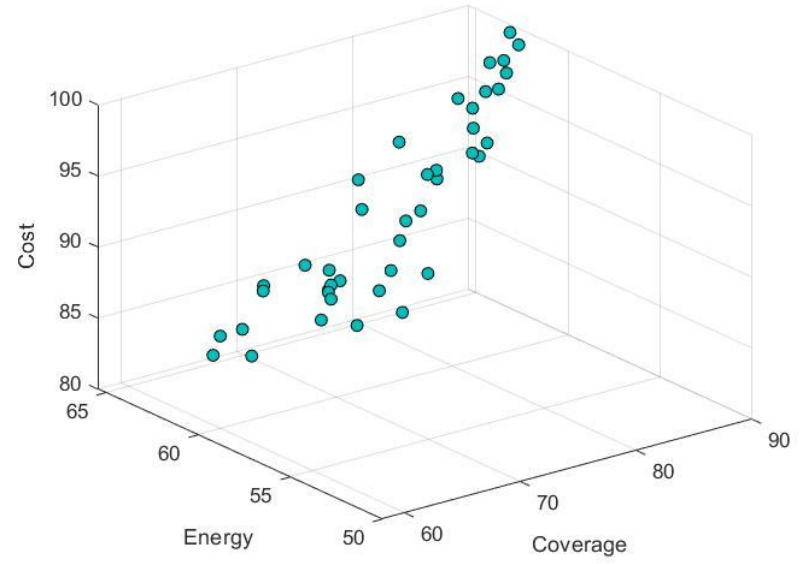

Figure 2: The Pareto Front for the Instance of size $600 \times 600 \mathrm{~m}^{2}$

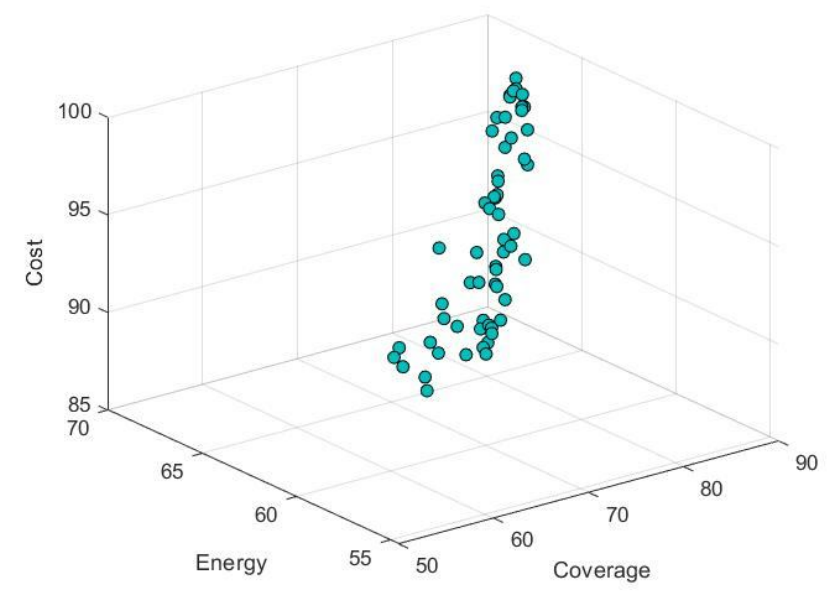

Figure 3: The Pareto Front for the Instance of size $800 \times 800 \mathrm{~m}^{2}$

\subsection{Results and Discussion}

The collected results indicating the solution quality based on the main considered optimization objectives are listed in Table 2 for all the experiments. It is evident that the proposed GP-driven greedy-enhanced GP optimization model provided a highly comparable solution quality to that of the multiobjective GA model. In the case of Exp1 where the network coverage was more preferable, for example, both models achieved a high rate of more than $92 \%$. In another example where minimizing energy consumption was prioritized, the rate was minimized to less than $55 \%$ by both models in Exp6. It was also achievable to reduce deployment cost by more than $12 \%$ in all the experiments where the cost objective was given higher weight value by AHP (Exp2, Exp5, Exp8). All these achievements were made possible by the proposed approach irrespective of the scale of the RNP problem.

Table 2: Results of the Proposed GP-driven greedy-enhanced GA Model

\begin{tabular}{|c|c|c|c|}
\hline Exp. & $\begin{array}{c}\text { Network } \\
\text { Coverage } \\
\text { Rate }\end{array}$ & $\begin{array}{c}\text { Energy } \\
\text { Consumption } \\
\text { Rate }\end{array}$ & $\begin{array}{c}\text { Cost } \\
\text { Rate }\end{array}$ \\
\hline Exp1 & 95.2 & 55.8 & 96.0 \\
\hline Exp2 & 84.8 & 61.5 & 86.0 \\
\hline Exp3 & 83.2 & 51.2 & 88.0 \\
\hline Exp4 & 92 & 59.9 & 96.82 \\
\hline Exp5 & 86.7 & 62.8 & 85.7 \\
\hline Exp6 & 81.8 & 52.8 & 95.2 \\
\hline Exp7 & 83.0 & 63.7 & 97.8 \\
\hline Exp8 & 76.8 & 64.2 & 86.8 \\
\hline Exp9 & 73.5 & 53.2 & 97.8 \\
\hline
\end{tabular}


Moreover, our proposed model was able to achieve relatively better solution quality compared to the multiobjective GA model. This is more evident in all the experiments with relatively small-scale RNP problem (Exp1-6). For instance, it is clear in Exp5 that all the performance considering all the objectives were improved by $2-13 \%$. In another example, improvements of $3-12 \%$ on all the rates were achieved by the proposed model in Exp3. It can be seen that our GP-based optimization approach was able to even improve the solution quality provided by the multiobjective GA model as the problem scale is relatively small. However, it still provides a highly comparable solution quality to that of the multiobjective GA model as the problem scales up.

\section{CONCLUSION}

A mathematical model which optimizes multiple objectives for effective deployment of energy-harvesting RNs in pre-established stationary WSNs was introduced in this paper. The focus was on maximizing network coverage, improving energy efficiency, and minimizing deployment cost. To this end, a novel Goal Programming (GP)-driven optimization approach was proposed. Problem formulation was achieved using the weighted-sum GP model integrated with an Analytical Hierarchy Process (AHP) method for effectively assigning weights to the decision variables. A GA model enhanced with a greedy technique was then developed to solve the GP-formulated RNP problem. To evaluate our novel approach, experimental investigation of the effectiveness of the AHP technique in generating the parameters for the GP model was carried out. It is evident from the evaluation results that, our GP-driven greedy-enhanced GP optimization model provided a highly comparable solution quality to that of the multiobjective GA model. It also was able to outperform the multiobjective GA model in the relatively small-scaled RNP problems, considering all the different combinations of the AHP-assigned weights. As a future research work, we can validate our integrated approach further by applying it for some other multi-objective optimization problems that have more conflicting objectives. In such cases, applying AHP to generate weights would become more challenging. We then suggest to check the validity of AHP model carefully by comparing between Consistency Index and Random Consistency Index.

\section{ACKNOWLEDGEMENT}

This work was supported by Deanship of Scientific Research, Qassim University, according to the agreement of the funded project No. coc-2018-1-14-S-5014. The author thanks the sponsor of this work for their support.

\section{REFERENCES}

1. Z. Fei, B. Li, S. Yang, C. Xing, H. Chen, and L. Hanzo. A survey of multi-objective optimization in wireless sensor networks: Metrics, algorithms, and open problems, IEEE Commun. Surveys Tuts., vol. 19, no. 1, pp. 550-586, 2016.

2. M. Iqbal, M. Naeem, A. Anpalagan, A. Ahmed, and M. Azam. Wireless sensor network optimization: Multi-objective paradigm, Sensors, vol. 15, no. 7, pp. 17572-620, July 2015.

3. M. Iqbal, M. Naeem, A. Anpalagan, N.N. Qadri, and M. Imran. Multi-objective optimization in sensor networks: Optimization classification, applications and solution approaches, Comput. Networks., vol. 99, pp. 134-161, 2016.

4. S. Amaran and R. M. Mohan. An Optimal Grey Wolf Optimization with Fuzzy Support Vector Machine based Intrusion Detection System in Clustered Wireless Sensor Networks, International Journal of Advanced Trends in Computer Science and Engineering, vol. 9, no. 3, pp. 2655-2661, June 2020.

5. B. M. Nezhad, M. Alizadeh, T. Khodadadi. Extending the Network Lifetime by Distributed Selection of Cluster Head in Homogeneous Wireless Sensor Networks, International Journal of Advanced Trends in Computer Science and Engineering, vol. 9, no. 3, pp. 3107-3112, June 2020.

6. I. F. Akyildiz, W. Su, Y. Sankarasubramaniam, and E. Cayirci. Wireless sensor networks: a survey, Computer networks, vol. 38, no. 4, pp. 393-422, Mar 2002.

7. J. M. Lanza-Gutiérrez, N. Caballé, J. A. Gómez-Pulido, B. Crawford, and R. Soto. Toward Robust Multi-Objective Metaheuristic for Solving the Relay Node Placement Problem in Wireless Sensor Networks, IEEE Sensors J., vol. 19, no. 3, pp. 677, 2019.

8. J. P. Ignizio and C. Romero. Goal programming, Encycl. Inf. Syst, vol. 2, pp. 888-898, 2003.

9. A. Charnes and W. W. Cooper. Goal programming and multiple objective optimizations. Eur. J. Oper. Res., vol. 1, pp. 39-54, 1977.

10. M. Tamiz, D. Jones, and C. Romero. Goal programming for decision making: An overview of the current state-of-the-art. Eur J Operation Res, vol. 111, no. 3, pp 567-581, 1998.

11. J. P. Ignizio. Introduction to linear goal programming, Beverly Hills, CA.: Sage; 1985.

12. S. M. Lee. Goal programming for decision analysis, Philadelphia, PA: Auerbach; 1972.

13. C. T. Chang. Revised multi-choice goal programming, Applied Mathematical Modelling, vol. 32, no. 12, pp. 2587-2595, 2008.

14. A-J. Garcia-Sanchez, F. Garcia-Sanchez, D. Rodenas-Herraiz, and J. Garcia-Haro. On the Optimization of Wireless Multimedia Sensor Networks: A Goal Programming Approach, Sensors, vol. 12, no. 9 pp. 12634-12660, 2012.

15. S. A. Khan and M. Baqer. Goal programming approach for multi-criteria decision-making for an energy efficient event recognition scheme, in Proc. IEEE Symposium on Computational Intelligence in Multi-Criteria Decision-Making (MCDM), Singapore, 2013, pp. 56-60.

16. R. Y. AlOmary and S. A. Khan. Goal programming based multi-criteria decision-making for distributed 
denial of service attacks in wireless sensor networks, in Proc. International Conference on Computer, Control, Informatics and Its Applications (IC3INA), Jakarta, 2013, pp. 33-37.

17. D. Rodenas-Herraiz, A. Garcia-Sanchez, F. Garcia-Sanchez, and J. Garcia-Haro. On the improvement of wireless mesh sensor network performance under hidden terminal problems, Future Generation Computer Systems, vol. 45, pp. 95-113, 2015.

18. X. Zheng and C. Chang. Topology design of remote patient monitoring system concerning qualitative and quantitative issues, Omega, 102137, 2019.

19. R. Arab Loodaricheh, S. Mallick, and V. K. Bhargava. Resource allocation with QoS provisioning for energy harvesting systems: A goal programming approach, in Proc. IEEE International Conference on Communications (ICC), London, 2015, pp. 2791-2796.

20. C. S. Wang and C. T. Chang. Goal-programming-driven genetic algorithm model for wireless access point deployment optimization, Mathematical problems in engineering, vol. 2012, Article ID 780637, Jan 2012.

21. M. Azharuddin and P. K. Jana. A GA-based approach for fault tolerant relay node placement in wireless sensor networks, in Proc. The $3^{\text {rd }}$ International Conference on Computer, Communication, Control and Information Technology (C3IT), Hooghly, 2015, pp. 1-6.

22. T. L. Saaty. The Analytic Hierarchy Process. McGraw Hill, New York, 1980.

23. J. Zhang and R. Yan. Multi-objective Distributed Clustering Algorithm in Wireless Sensor Networks Using the Analytic Hierarchy Process, in Proc. the 20th IEEE/ACIS International Conference on Software Engineering, Artificial Intelligence, Networking and Parallel/Distributed Computing (SNPD), Toyama, Japan, 2019, pp. 88-93.

24. F. El Hajji, C. Leghris, and K. Douzi. Adaptive routing protocol for lifetime maximization in multi-constraint wireless sensor networks, Journal of Communications and Information Networks, vol. 3, no. 1, pp. 67-83, Mar 2018.

25. Y. Chang, H. Tang, B. Li, and X. Yuan. Distributed Joint Optimization Routing Algorithm Based on the Analytic Hierarchy Process for Wireless Sensor Networks, IEEE Communications Letters, vol. 21, no. 12, pp. 2718-2721, Dec 2017.

26. K. Deb. Non-linear Goal Programming Using Multi-Objective Genetic Algorithms, Journal of the Operational Research Society, vol. 52, no. 3, pp. 291-302, 2001. 